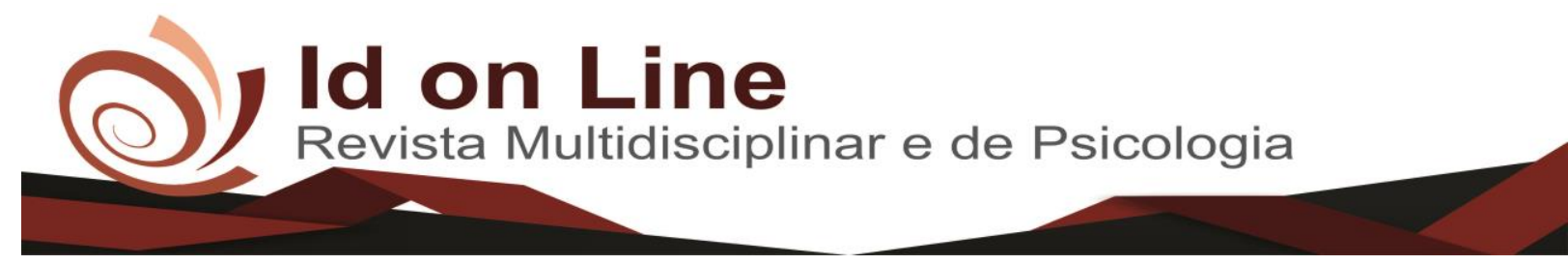

Artigo de Revisão

\title{
Doenças Bucais que mais acometem o paciente na terceira idade: Uma revisão de Literatura
}

\author{
Anna Luisa Albeny ${ }^{l}$; Débora Bittencourt Ferreira Santos ${ }^{2}$
}

\begin{abstract}
Resumo: O envelhecer é uma consequência natural do desenvolvimento sendo entendida por um conjunto de modificações morfológicas e fisiológicas. O surgimento de alterações que se manifestam na cavidade oral do idoso, revela a interação existente entre as consequências degenerativas da idade e condições patológicas. As modificações sofridas no sistema estomatognático dos pacientes geriátricos possuem grande frequência clínica e necessitam de conhecimentos aprofundados na literatura, de modo a fornecerem informações que contribuam para o aprimoramento deste campo da Odontologia. Objetivo: Este estudo teve como propósito abordar as principais alterações bucais que acometem o idoso destacando as causas, o diagnóstico e prevenção das mesmas. Conclusão: Verificou-se uma variedade de alterações que acometem a cavidade bucal de pacientes idosos como edentulismo, doença periodontal, cáries, lesões de mucosa oral e xerostomia. Nesse contexto, o cirurgião dentista tem papel fundamental na detecção dessas patologias para que haja um correto diagnóstico e tratamento das mesmas a fim de que possa contribuir para um envelhecimento saudável ao paciente geriátrico.
\end{abstract}

Palavras-chave: Idoso; Odontogeriatria; Patologia Oral.

\section{Oral Diseases that most affect the patient in the Elderly: A Literature Review}

\begin{abstract}
The aging is a natural consequence of the development being understood by a set of morphological and physiological changes. The emergence of changes that manifest in the oral cavity of the elderly, reveals the interaction that exists between the degenerative consequences of age and pathological conditions. The changes suffered in the Stomatognathic system of geriatric patients have clinical and often require a thorough knowledge in the literature, in order to provide information that will contribute to the improvement of this field of dentistry. 10 Objective: The purpose of this study was to address the main oral changes that affect the elderly, highlighting the causes, diagnosis and prevention. Conclusion: There was a variety of changes that affect the oral cavity of elderly patients as edentulous, periodontal disease, dental caries, lesions of the oral mucosa and xerostomia. In this context, the dentist has a fundamental role in the detection of these diseases so that there is a correct diagnosis and treatment of the same so that can contribute to a healthy ageing to the geriatric patient.
\end{abstract}

Keywords: Elderly; Geriatric dentistry; Oral Pathology.

\footnotetext{
${ }^{1}$ Graduanda em Odontologia, pela Faculdade Independente do Nordeste - FAINOR, Vitória da Conquista, Bahia, Brasil. Contato: annalualbeny@gmail.com;

${ }^{2}$ Docente do curso de Odontologia da Faculdade Independente do Nordeste - FAINOR; Cirurgiã-dentista pela UNIVALE Minas Gerais; Especialista em ortodontia pela SL MANDIC - Campinas; Mestre na área de concentração ortodôntica pela SL MANDIC - Campinas.
}

681 Id on Line Rev. Mult. Psic. V.12, N. 42, p. p. 681-694, 2018 - ISSN 1981-1179 Edição eletrônica em http://idonline.emnuvens.com.br/id 


\section{Introdução}

No decorrer dos últimos anos a população mundial tem passado por um aumento significativo na quantidade de idosos, principalmente nos países em desenvolvimento, devido um aumento na expectativa média de vida (CATÃO, XAVIER e PINTO, 2011). A população brasileira vem envelhecendo e os vários setores da sociedade assim como os profissionais da saúde devem se preparar para dar suporte e contribuir para uma maior qualidade de vida deste crescente grupo (AUSTREGÉSILO, LEAL, MARQUES, VIEIRA E, ALENCAR, 2015).

No processo de envelhecimento o corpo humano sofre alterações fisiológicas consideráveis, sendo necessário que o Cirurgião-Dentista tenha ciência dessas mudanças uma vez que a maioria delas acometem a saúde bucal (CÔRTE-REAL, FIGUEIRAL e CAMPOS, 2015). O elevado número de indivíduos idosos, a particularidade da sua situação dentária, são fatores que aumentam o impacto no tratamento dentário sendo necessário, portanto, uma abordagem multidisciplinar no atendimento destes pacientes, e esse fato tem sido um desafio na formação de profissionais que possuam tais características (SOUSA et al, 2013).

O avançar da idade promove um aumento na prevalência de algumas doenças bucais, visto que a mucosa oral torna-se mais susceptível aos danos mecânicos (VACCAREZZA, FUGA e FERREIRA, 2010). Algumas doenças sistêmicas e determinados medicamentos apresentam efeitos colaterais na secreção salivar, gerando na maioria das vezes um processo de hipossalivação que aumenta desta forma o risco de surgimento de lesões intrabucais na população geriátrica (BARBOSA, 2011).

Sendo assim, o Cirurgião-Dentista que se preocupa com o bem-estar do seu paciente, e sendo este idoso, deve procurar instruir-se sobre os cuidados específicos que a população idosa necessita. Além disso, deve entender que o atendimento deve ser empregado por meio de uma visão ampla, visto que, na maioria das vezes, esta população traz consigo um conjunto de patologias sistêmicas que interferem na saúde bucal (SOUSA et al, 2013).

Dentre as principais doenças orais que acometem a cavidade oral com o avançar da idade pode-se destacar a xerostomia, a perda da capacidade gustativa, doenças periodontais, cárie dentária oriunda da dificuldade motora no processo de higienização, que podem evoluir para perdas dentárias e algumas lesões na mucosa acarretando assim uma diminuição na 
qualidade de vida dos idosos (CATÃO, XAVIER e PINTO, 2011; AUSTREGÉSILO, LEAL, MARQUES, VIEIRA e ALENCAR, 2015; CÔRTE-REAL, FIGUEIRAL e CAMPOS; SOUSA et al, 2013).

Sendo assim, o Cirurgião-Dentista deve se preocupar com o bem-estar do seu paciente como um todo, e tratando-se de um idoso, o profissional deve procurar instruir-se sobre os cuidados específicos que essa faixa etária necessita. Além disso, deve entender que o atendimento deve ser empregado por meio de uma visão ampla, visto que, na maioria das vezes, esta população traz consigo um conjunto de patologias sistêmicas que interferem na saúde bucal (SOUSA et al, 2013).

É fundamental a criação de um plano de ação condizente com a saúde bucal e as necessidades de tratamento deste grupo. Esse plano de ação deve conter serviços de saúde selecionados para esta faixa etária, incluindo atividades educacionais permanentes, procedimentos preventivos, restauradores e de reabilitação (VACCAREZZA, FUGA e FERREIRA, 2010). A fim de se obter maior efetividade nas ações de saúde bucal para os idosos, é importante o avaliar a autopercepção que estes pacientes têm em relação aos impactos das condições de saúde bucal na sua qualidade de vida (BARBOSA, 2011).

A atenção médica e odontológica para esta população deve estar centralizada essencialmente na melhoria da qualidade de vida, com especial atenção à prevenção de doenças e outras disfunções (BARBOSA, 2011). A saúde bucal, parte integrante e inseparável da saúde geral dos indivíduos, algumas vezes são tratadas com descaso, devido o fato de grande parcela desse grupo ser edêntula (ROSENDO et al, 2017; SILVA et al, 2011).

Tendo em vista esse envelhecimento da população e o fato do crescente número de idosos na sociedade, que por sua vez apresentam características peculiares e doenças específicas deste grupo, o presente trabalho tem o objetivo de abordar as principais alterações e as doenças bucais que acometem os pacientes dessa faixa etária, dando ênfase as manifestações clínicas, o seu diagnóstico e medidas de prevenção das mesmas. 


\section{Materiais e métodos}

A metodologia para ser realizada a elaboração deste artigo será a revisão de literatura, buscando uma síntese do conhecimento acerca das principais afecções bucais que mais acometem os pacientes da terceira idade.

Para tanto serão utilizadas as seguintes palavras-chaves: Idoso; Odontogeriatria; Patologia Oral, e suas respectivas em inglês, para acesso às bases de dados Literatura LatinoAmericana e do Caribe em Ciências e Saúde (LILACS), Pubmed e Scielo.

O período a ser pesquisado compreende de 2008 a 2018, com intuito de ter acesso aos trabalhos mais recentes sobre o assunto em questão.

Serão incluídos somente artigos científicos, dentro da temática proposta, selecionado por meio do título e do resumo, com textos completos, publicados na língua portuguesa e inglesa. Por sua vez, serão adotados os seguintes critérios de exclusão: trabalho em outro formato que não artigo, como teses e dissertações, os que fogem da temática proposta, artigos incompletos e/ou publicados em outro idioma que não os citados anteriormente.

\section{Revisão de Literatura}

\section{Principais alterações bucais relacionadas ao processo de envelhecimento}

Grande parte das alterações bucais, teciduais e funcionais que acometem o paciente idoso está relacionada com fatores extrínsecos e intrínsecos que ocorrem no decorrer da vida, portanto, quando há preservação, as alterações das funções orais decorrentes da idade se tornam mínimas (ROSENDO et al, 2017; SILVA et al, 2011; FERNANDES-COSTA et al, 2013; CARVALHO, MANSO, ESCOVAL, SALVADO e NUNES, 2013). A negligência nos procedimentos de higiene oral bem como as visitas não periódicas aos consultórios odontológicos, e as alterações fisiológicas, como a perda da elasticidade da mucosa, sofridas pelos idosos são fatores que podem promover o desenvolvimento de afecções bucais (VASCONCELOS, 2012). 
A maioria dos problemas odontológicos observados na população da terceira idade está relacionada com complicações patológicas adquiridas no decorrer da vida destes pacientes, oriundas de uma ineficiente higiene bucal, iatrogenia de profissionais, falta de orientação, e de acesso aos serviços de saúde, seja por negligência pessoal ou condição socioeconômica (LELIS et al, 2009).

Dentre as alterações percebidas na cavidade oral do paciente idoso algumas decorrentes manifestações de doenças sistêmicas, deficiência nutricional, efeito colateral de determinados medicamentos, que repercutem no funcionamento das glândulas salivares, na saúde do tecido periodontal, e da mucosa oral (ROLDAN, HERRERA e SANZ, 2013). Destas o edentulismo, a cárie dentária, doença periodontal, a xerostomia, redução da capacidade gustativa, e as lesões nos tecidos moles são as que apresentam maior prevalência e acometimento na saúde bucal do idoso (CATÃO, XAVIER e PINTO, 2011; AUSTREGÉSILO, LEAL, MARQUES, VIEIRA E, ALENCAR, 2015).

\section{Edentulismo}

Caracterizado por perda dos elementos dentários, acarretando ao paciente uma série de problemas no quesito funcional, psicológico e social, refletindo de maneira direta na alimentação do paciente bem como em sua qualidade de vida (SOUSA et al, 2013). É uma condição oral de alta prevalência entre os idosos. Dentre as principais causas relacionadas com essa perda pode-se destacar o consumo de tabaco (CATÃO, XAVIER e PINTO, 2011; SILVA et al, 2011), etilismo, ineficiência nas práticas de higiene oral, longos períodos de internação hospitalar, e o baixo nível socioeconômico que na maioria das vezes leva a uma baixa frequência de consultas odontológicas (CATÃO, XAVIER e PINTO, 2011; SOUSA et al, 2013;).

Alguns estudos relacionam a ausência total de dentes a pacientes com idade mais avançadas, uma vez que estes em conjunto com o processo de envelhecimento aumentam a vulnerabilidade destes pacientes para o desenvolvimento de determinadas doenças bucais (SHIP et al, 2013). Muitos desses problemas que acometem a população idosa são, na realidade, 
complicações de processos patológicos acumulados no decorrer da vida do indivíduo (LELIS et al, 2009).

A falta total de dentes ainda é aceita pela sociedade como algo normal e natural com o avançar da idade, e não como reflexo da falta de programas preventivos de saúde bucais (ROCHA e MIRANDA , 2013; MORAES, ALBUQUERQUE e CHEVITARESE, 2017). A falta dos dentes leva o paciente a manter uma dieta mais pastosa, rica em carboidratos fato este que proporciona um aumento do peso corporal, e consequentemente pode levar ao desenvolvimento de doenças sistêmicas como a obesidade, a hipertensão arterial, diabetes entre outras (CARVALHO et al, 2013)

\section{Cárie Dentária}

Dados mundiais revelam que a cárie é a doença bucal mais comum entre os indivíduos com sessenta anos ou mais. Fatos como a redução no fluxo salivar decorrente o uso de determinados medicamentos, dificuldade motora no processo de higienização e as alterações na dieta potencializam de maneira direta os efeitos dessa doença (FERNANDES-COSTA et al, 2013). Dentre as principais causas que levam a perda dentária a cárie é considerada o principal fator de risco associado à má higiene bucal (CARVALHO et al, 2013).

Em consequência da limitação da função motora e mental dos idosos, há uma limitação no processo de higienização bucal facilitando dessa forma o aumento no acúmulo de biofilme dental que por sua vez ocasionam o surgimento de lesões cariosas, estas podem evoluir levando à deterioração dos tecidos periodontais, predispondo o indivíduo ao surgimento de cáries radiculares (VACCAREZZA, FUGA e FERREIRA, 2010; ROSENDO et al, 2011).

\section{Doença periodontal}

Assim como o processo carioso, a doença periodontal, é decorrente ao acúmulo da placa bacteriana na superfície dos dentes, acarretando por sua vez em destruição dos tecidos adjacentes. Os metabólitos bacterianos adentram os tecidos periodontais promovendo uma resposta inflamatória exacerbada. Essas mudanças estão relacionadas com a diminuição da 
destreza manual, a redução no sistema de defesa imunológico (SILVA et al, 2011) destes pacientes, e o processo de envelhecimento celular do periodonto que torna o processo cicatrizacional mais demorado (CÔRTE-REAL, FIGUEIRAL e CAMPOS, 2011; VASCONCELOS, 2012; LELIS et al, 2009).

Mudanças ocorridas na dieta e uma diminuição na quantidade de saliva promovem um acúmulo mais rápido de biofilme na superfície dentária de pacientes idosos (CATÃO, XAVIER e PINTO, 2011). O avançar da idade também promove uma alteração gradual no tecido ósseo, com diminuição do tecido mineralizado, onde a reabsorção aumenta e a neoformação óssea diminui, resultando em um aumento na porosidade óssea (AUSTREGÉSILO, LEAL, MARQUES, VIEIRA E, ALENCAR, 2015; VACCAREZZA, FUGA e FERREIRA, 2010; BARBOSA, 201).

A periodontite crônica com o avançar da idade mostra-se mais severa, uma vez que fatores sistêmicos e a condição de saúde geral do paciente influenciam na progressão da doença (ROSENDO et al, 2017; SILVA et al, 2011). Situações como uma quantidade reduzida de dentes, doenças sistêmicas, tabagismo, etilismo, associadas à ausência de acompanhamento odontológico contribuem de maneira direta para a progressão da doença periodontal (MORAES, ALBUQUERQUE e CHEVITARESE, 2017).

Como consequência a doença periodontal promove a perda dentária, diminuindo a capacidade mastigatória do paciente, repercutindo de forma direta nas funções do sistema estomatognático, além do desenvolvimento de halitose, que interfere na relação social deste com os indivíduos que convive (VASCONCELOS, 2012).

\section{Xerostomia}

O paciente idoso sofre uma perda de $30 \%$ da capacidade funcional das glândulas salivares, havendo consequentemente uma diminuição no processo de lubrificação da cavidade oral, tornando o indivíduo mais susceptível ao desenvolvimento de condições patológicas. Aproximadamente $40 \%$ dos idosos que frequentam os consultórios odontológicos se queixam da xerostomia, que afeta de maneira direta a fala, mastigação e deglutição destes pacientes, podendo gerar até mesmo episódios dolorosos (SILVA et al, 2008). 
Dentre os medicamentos que contribuem para desenvolvimento desse quadro destacamse os anti-hipertensivos, ansiolíticos, anti-depressivos, anti-histamínicos e anticolinérgicos; também existem procedimentos específicos que tornam os idosos mais susceptíveis a esta alteração, como a radioterapia utilizada no tratamento do câncer (SHIP et al, 2013). Outro fator contribuinte é o hábito tabagista (CATÃO, XAVIER e PINTO, 2011), uma vez que este em conjunto com a disfunção glandular aumenta a incidência de gengivite, o risco de candidíase oral, além de dificultar a mastigação, deglutição, gustação, fala e diminuição da retenção protética (CATÃO, XAVIER e PINTO, 2011; VASCONCELOS, 2012; PRESA e MATOS, 2014).

\section{Redução da capacidade gustativa}

Aproximadamente $80 \%$ dos idosos apresentam uma redução da capacidade gustativa decorrente uma diminuição na quantidade de papilas gustativas, esse decréscimo ocorre significativamente após os 70 anos de idade (ROSENDO et al, 2017; ROLDAN, HERRERA e SANZ, 2013).

A saburra lingual ocorre devido ao acúmulo de restos alimentares e bactérias no dorso da língua, promovendo também uma diminuição gustativa. A presença dessa saburra pode estar relacionada com a halitose, e a pneumonia aspirativa (ROLDAN, HERRERA e SANZ, 2013).

A higienização do dorso da língua, seja ela com escova dental ou gaze é uma prática preventiva na diminuição dos sintomas da redução gustativa (ROSENDO et al, 2017).

\section{Lesões da mucosa oral}

Alterações metabólicas que ocorrem no processo de envelhecimento, como a podem gerar a perda da morfologia gengival, tornando a mucosa oral atrofiada e friável. Essas alterações resultam na diminuição da capacidade de recuperação dos tecidos bucais e redução do suprimento sanguíneo prejudicando dessa forma o processo de regeneração tecidual (ROLDAN, HERRERA e SANZ, 2013). 
A estomatite protética causada por desadaptação protética, é uma afecção de alta prevalência clínica e apresentada com grande frequência por pacientes idosos (LELIS et al, 2009). Outras lesões que podem ser observadas em pacientes que fazem uso de prótese são a hiperplasia fibroepitelial, úlcera traumáticas e a queilite angular (PINZÓN-PULIDO e GILMONTOYA, 2009).

O câncer bucal é uma doença que afeta, em sua maioria, pacientes de sexo masculino, que se encontram entre os 50 e 60 anos de vida, e que apresentam o hábito tabagista e etilista. A incidência dessa doença traz complicações severas, sendo responsável direta por elevadas taxas de mortalidade (SILVA et al, 2008; PINZÓN-PULIDO e GIL-MONTOYA, 2009). O autoexame aliado ao diagnóstico precoce são métodos preventivos que requerem um envolvimento multidisciplinar, incorporando cirurgiões-dentistas, médicos, enfermeiros e nutricionistas (SILVA et al, 2008).

\section{Atendimento odontológico para pacientes geriátricos}

O tratamento odontológico a ser realizado com esse tipo de paciente deve levar em conta o diálogo com o mesmo e a análise da sua percepção da condição bucal sempre que possível, por meio de conversação com os familiares e/ou cuidadores no momento do preenchimento da anamnese é de suma importância, pois o profissional fica ciente do estado de saúde geral deste paciente, bem como os fármacos que o mesmo faz uso, além das suas expectativas frente ao tratamento (SOUSA et al, 2013; ROSENDO et al, 2017; FERNANDES-COSTA ET AL, 2013).

O tratamento preventivo do idoso deve ser realizado com auxílio multiprofissional, buscando uma maior percepção, de todos os profissionais envolvidos no cuidado desses pacientes, acerca da condição de saúde geral do mesmo, visando diminuir os problemas de saúde e evitando dessa forma possíveis intervenções que tragam algum prejuízo a estes (ROLDAN S, HERRERA e SANZ, 2013).

Cabe ao Cirurgião-Dentista a função de identificar e analisar questões específicas da condição de saúde bucal do paciente geriátrico, visando realizar ações preventivas que consigam atingir ao máximo o seu público, através de palestras educativas, orientações de 
higiene oral, e da necessidade da participação dos familiares e/ou cuidadores no processo de atenção à saúde do idoso (VACCAREZZA, FUGA e FERREIRA, 2010).

\section{Discussão}

O crescimento da população idosa, observado principalmente nos países desenvolvidos, ocorre devido um aumento na expectativa e qualidade de vida destes. O estágio na velhice apesar de não ser sinônimo de doença é sabido que há uma diminuição significativa na capacidade funcional do indivíduo, tornando-o cada vez mais dependente com o avançar da idade (CATÃO, XAVIER e PINTO, 2011).

De acordo com Silva et al (2011), o processo de envelhecimento não está estritamente relacionado com a perda ou diminuição das funções corpóreas; entretanto Vasconcelos (2012) afirmar que com o decorrer dos anos o corpo humano sofre mudanças tanto físicas, quanto mentais, sociais e comportamentais, que por sua vez levam o paciente idoso a desenvolver determinadas enfermidades, peculiares da idade. $\mathrm{O}$ avançar da idade traz também alterações na cavidade oral destes pacientes, sendo a maioria delas consequência do estado geral de saúde, bem como efeito colateral de alguns medicamentos, dificuldade motora na realização da higiene bucal, e acesso pouco frequente aos cuidados preventivos (CATÃO, XAVIER e PINTO, 2011).

Algumas doenças sistêmicas acometem com maior frequência indivíduos da terceira idade dentre elas destacam a hipertensão, cardiopatias, diabetes, artrite e bronquite, e frequentemente, esses pacientes fazem uso crônico de medicação para controle dessas enfermidades, que em determinados casos podem apresentar acometimento direto da mucosa oral e elementos dentários (SOUSA et al, 2013).

Para correto diagnóstico é necessário que o profissional tenha conhecimento das estruturas normais bucais e suas variações anatômicas. De acordo com Lelis e colaboradores (2009) é comum os idosos apresentarem problemas odontológicos, dentre estes destacam: a perda dos elementos dentários que pode levar ao edentulismo total, doença no complexo periodontal, cáries, alterações nas mucosas bucal e lingual, diminuição da atividade das glândulas salivares, e a incidência de neoplasias malignas. 
A falta de medidas preventivas no passado determinou a condição bucal do idoso atualmente, onde o edentulismo tem uma alta prevalência. Estudos realizados por PinzónPulido e Gil-Montoya (2009) observaram que cerca de 30\% da população idosa é desdentada, e essa condição tem predominância no sexo feminino. Presa (2014) afirma que o hábito tabagista aumenta o desenvolvimento desse quadro.

O principal fator para pré-disposição ao desenvolvimento da cárie e da doença periodontal, é o acúmulo de biofilme na superfície dentária, onde Roldan, Herrera e Sanz (2013) em seu estudo após a realização dos exames clínico e periodontal, em idosos, observou a presença de placa bacteriana, e higiene bucal deficiente, justificando dessa forma a condição patológica destes pacientes.

Fernandes-Costa e colaboradores (2013) relatam que mudanças como a diminuição na destreza manual, decréscimo da capacidade das células de defesa, alterações na composição e textura da dieta e a diminuição do fluxo salivar, favorecem o acúmulo mais rápido do biofilme nas superfícies dentárias dos pacientes idosos, tornando-os mais susceptíveis ao desenvolvimento da cárie e doença periodontal.

No que se refere ao fluxo salivar dos idosos, os autores mostram-se controvérsia, uma vez que para Barbosa (2011), a minimização deste fluxo na terceira idade é gradativamente diminuído não gerando assim nenhum efeito significativo. Entretanto Roldan, Herrera e Sanz (2013) afirmam que o fluxo salivar se mantém inalterado no decorrer da vida. E em contrapartida Austregésilo et al. (2015), acredita que a diminuição na excreção das glândulas salivares pode estar diretamente relacionada com o uso de determinados medicamentos e/ou presença de alguma enfermidade sistêmica, afetando de forma significativa a saúde bucal do idoso.

Sousa e colaboradores (2013) em seu estudo observaram que a diminuição da capacidade gustativa acomete aproximadamente $80 \%$ dos indivíduos que participaram da pesquisa. Essa perda gustativa pode ser associada à deficiente higienização bucal que promove, por sua vez, à formação da saburra lingual. Corroborando com este estudo Vaccarezza, Fuga e Ferreira (2010), constataram que a melhora da higienização promoveu um aumento na percepção de doce e salgado por esses pacientes.

Um estudo com idosos constatou que $70 \%$ faziam uso de próteses, e dentre esses foi observado um elevado número de candidíase, periodontite severa e hiperplasia do palato, 
devido uso da prótese, essa alta incidência se dá principalmente devido ao uso de peças mal adaptados e falta de orientação dos profissionais da necessidade de higienização e manutenção dessas próteses (ROSENDO et al, 2017).

Há uma série de lesões orais que podem se desenvolver decorrente o uso de próteses removíveis, sendo as estomatites, úlceras por trauma, lesões periodontais, candidíase e hiperplasias as que surgem com maior frequência (SHIP et al, 2013). Autores (CÔRTE-REAL, FIGUEIRAL e CAMPOS, 2011) sugerem que algumas lesões orais podem tornar-se malignas devido traumatismo crônico gerados pela má adaptação protética. Em contrapartida Silva et al (2011) acreditam que o trauma por si só não desencadeia o câncer bucal.

A Odontogeriatria foi recentemente incorporada aos cursos de graduação na Odontologia, e reconhecida como especialidade, entretanto, há uma gama de desafios a serem vencidos, uma vez que, a saúde bucal muitas vezes não é reconhecida como parte integrante da saúde geral do indivíduo, sendo muitas vezes negligenciada tanto pelo paciente quanto por demais profissionais da área de saúde (CARVALHO et al, 2013; ROCHA e MIRANDA, 2013; MORAES, ALBUQUERQUE e CHEVITARESE, 2017).

O preenchimento correto e completo da anamnese durante as consultas médicas e o encaminhamento dos pacientes idosos ao profissional Odontogeriátrico mostram que um atendimento multidisciplinar traz resultados satisfatórios à prevenção e cura das enfermidades que acometem o paciente na terceira idade (SILVA et al, 2008).

\section{Conclusões}

De acordo com a bibliografia consultada pode-se concluir que há uma gama de alterações bucais que acometem o paciente na terceira idade, como o edentulismo, cárie, doença periodontal, a redução da capacidade gustativa, xerostomia e lesões orais.

Cabe ao Cirurgião-Dentista o papel de ofertar ao paciente idoso um tratamento diferenciado, sugerindo meios de prevenção dessas afecções bucais, além de avaliar melhores formas de conduzir o tratamento, visando que o benefício trazido com o mesmo superem os danos, e orientá-los sobre a necessidade da higienização bucal, independentemente da quantidade de elementos dentários presentes em boca. 


\section{Referências}

AUSTREGÉSILO SC, LEAL MCC, MARQUES APO, VIEIRA JCM, ALENCAR DL. Acessibilidade a serviços de saúde bucal por pessoas idosas: uma revisão integrativa. Rev Bras Geriatr Gerontol 2015; 18(1):189-99.

BARBOSA KGN. Condições de saúde bucal em idosos: uma revisão da realidade brasileira. Rev Odontol Clín-Cient Recife 2011; 10(3):227-31.

CARVALHO C, MANSO AC, ESCOVAL A, SALVADO F, NUNES C. Tradução e validação da versão portuguesa do Geriatric Oral Health Assessment Index (GOHAI). Rev Port Saúde Pública 2013; 31 (2):166-72.

CATÃO MHCV, XAVIER AFC, PINTO TCA. O impacto das alterações do sistema estomatognático na nutrição do idoso. Rev Bras de Ciên da Saúd 2011; 29(9):73-8.

CÔRTE-REAL IS, FIGUEIRAL MH, CAMPOS JCR. As doenças orais no idoso considerações gerais. Rev Port Estomatol Med Dent Cir Maxilofac 2011; 52(3):175- 80.

FERNANDES-COSTA AN, VASCONCELOS MG, QUEIROZ LMG, BARBOZA CAG, VASCONCELOS RG. As Principais Modificações Orais que ocorrem durante o Envelhecimento. Rev Brasileira de Ciências da Saúde 2013; 17(3): 293-300.

LELIS ER, ROCHA ES, MATTAR DDS, MARTINS CR, SILVA DD, SOUSA DE SOUSA MLR, et al. Incidência e prevalência de doenças bucais em pacientes idosos: Alterações morfológicas, sistêmicas e bucais. Rev Inpeo Odontol 2009; 3(2):82-47.

MORAES CV, ALBUQUERQUE LC, CHEVITARESE L. A importância da Odontogeriatria para a oferta de cuidados bucais em idosos. Rev Acadêmica Rede de Cuidados em Saúde, 2017; 10(1): capa

PINZÓN-PULIDO SA, GIL-MONTOYA JA. Validación del índice devaloración de salud oral en geriatría en una población geriátrica institucionalizada de Granada. Rev Esp Geriatr Gerontol 2009; 34 (12):273-82.

PRESA SL, MATOS JC. Oral health in third age. Revista UNINGÁ, Maringá - PR, 2014; 39: $137-48$.

ROCHA DA, MIRANDA AF. Atendimento odontológico domiciliar aos idosos: uma necessidade na prática multidisciplinar em saúde: revisão de literatura. Rev. bras. geriatr. gerontol. $2013 ; 16(1)$ : 181-9

ROLDAN S; HERRERA D; SANZ M. Biofilms and the tongue: therapeutical approaches for the control of halitosis. Clin Oral Invest 2013; 7: 189-97. 
ROSENDO RA et al. Saúde bucal e impacto na qualidade de vida em idosos. RSC online, 2017; 6(1):89-102.

SHIP, JA., et al. Old age in health and disease - lesions from the oral cavity. Oral Surg Med Oral Pathol 2013; 76: 40-4.

SILVA DD, HELD RB, TORRES SV, SOUSA MDAL, NERI AL, ANTUNES JL. Selfperceived oral health and associated factors among the elderly in Campinas, Southeastern Brazil, 2008-2009. Rev. Saúde Pública, 2011; 45 (6): 1145-53.

SILVA EMM, et al. Main oral alterations and diseases in elderly patient - a literature review. Odonto 2011; 19 (37): 39-47.

SILVA SO, ROSSIE HS, LIMA PA, LAMEY OS, VELOSO MS, SOUZA MAL, et al. Saúde bucal do idoso institucionalizado em dois asilos de Passo Fundo - RS. RGO 2008; 56(3):30330 .

SOUSA MNA, BEZERRA ALD, ASSIS EV, NÓBREGA CBC, PELINO JEP. Oral healt conditions in old age: older adult's perception. J Nurs UFPE 2013; 7(3):68-76.

VACCAREZZA GF, FUGA RL, FERREIRA SRP. Saúde bucal e qualidade de vida dos idosos. Rev Odontol Univ São Paulo 2010; 22(2):134-7.

VASCONCELOS LCA. Impacto da saúde bucal no cotidiano de idosos de um município de médio porte do nordeste brasileiro. In: Cad. Saúde Pública. Rio de Janeiro 2012, 6 (28).

\section{Como citar este artigo (Formato ABNT):}

ALBENY, Anna Luisa; SANTOS, Débora Bittencourt Ferreira. Doenças Bucais que mais acometem o Paciente na Terceira Idade: Uma Revisão de Literatura. Id on Line Rev.Mult. Psic., 2018, vol.12, n.42, p. 681-694. ISSN: 1981-1179.

Recebido: 04/10/2018;

Aceito: 17/10/2018 